\title{
Structure, activity and inhibition of human TMPRSS2, a protease implicated in SARS-
} CoV-2 activation

Bryan J. Fraser\#, Serap Beldar\#, Almagul Seitova, Ashley Hutchinson, Dhiraj Mannar, Yanjun Li, Daniel Kwon, Ruiyan Tan, Ryan P. Wilson, Karoline Leopold, Sriram Subramaniam, Levon Halabelian*, Cheryl H. Arrowsmith*, François Bénard*

\# equal contribution

*Corresponding

\section{ABSTRACT}

Transmembrane protease, serine 2 (TMPRSS2) has been identified as key host cell factor for viral entry and pathogenesis of SARS-coronavirus-2 (SARS-CoV-2). Specifically, TMPRSS2 proteolytically processes the SARS-CoV-2 Spike (S) Protein, enabling virus-host membrane fusion and infection of the lungs. We present here an efficient recombinant production strategy for enzymatically active TMPRSS2 ectodomain enabling enzymatic characterization, and the $1.95 \AA$ X-ray crystal structure. To stabilize the enzyme for co-crystallization, we pre-treated TMPRSS2 with the synthetic protease inhibitor nafamosat to form a stable but slowly reversible (15 hour half-life) phenylguanidino acyl-enzyme complex. Our study provides a structural basis for the potent but non-specific inhibition by nafamostat and identifies distinguishing features of the TMPRSS2 substrate binding pocket that will guide future generations of inhibitors to improve selectivity. TMPRSS2 cleaved recombinant SARS-CoV-2 S protein ectodomain at the canonical S1/S2 cleavage site and at least two additional minor sites previously uncharacterized. We established enzymatic activity and inhibition assays that enabled ranking of clinical protease inhibitors with half-maximal inhibitory concentrations ranging from $1.7 \mathrm{nM}$ to $120 \mu \mathrm{M}$ and determination of inhibitor mechanisms of action. These results provide a body of data and reagents to support future drug development efforts to selectively inhibit TMPRSS2 and other type 2 transmembrane serine proteases involved in viral glycoprotein processing, in order to combat current and future viral threats.

\section{SUMMARY PARAGRAPH}

Viruses hijack the biochemical activity of host proteins for viral invasion and replication. Transmembrane protease, serine-2 (TMPRSS2) is a surface-expressed protease implicated in the activation of influenza A, influenza B, and coronaviruses, including SARS-CoV-2, to drive efficient infection of the lungs ${ }^{1-5}$. TMPRSS2 is an attractive target for antiviral therapies, as inhibiting its proteolytic activity blocks efficient viral entry ${ }^{5,6}$. However, a structural and biochemical understanding of the protease has remained elusive and no selective inhibitors are available. We engineered on-demand activatable TMPRSS2 ectodomain and determined the 1.95 $\AA$ X-ray crystal structure of the stabilized acyl-enzyme after treatment with nafamostat, a protease inhibitor under investigation as a COVID-19 therapeutic. The structure reveals unique features of the TMPRSS2 substrate recognition pocket and domain architecture, and explains the potent, but nonselective inhibition by nafamostat. TMPRSS2 efficiently cleaved the SARS-CoV-2 S protein at the canonical S1/S2 site as well as two minor sites previously uncharacterized. We further established a robust enzymatic assay system and characterized inhibition by two additional clinical protease inhibitors under study for COVID-19, camostat and bromhexine. Our results provide a body of data and reagents to enable ongoing drug development efforts to selectively inhibit TMPRSS2 and other TTSPs involved in viral glycoprotein processing, in order to combat current

46 and future viral threats. 


\section{MAIN}

\section{Production and structure of on-demand activatable TMPRSS2 ectodomain}

50 TMPRSS2 is a type 2 transmembrane serine protease (TTSP) comprised of an intracellular 51 domain, single-pass transmembrane domain, and a biologically active ectodomain with three 52 subdomains: a low density lipoprotein receptor type-A (LDLR-A) domain, a Class A Scavenger

53 Receptor Cysteine-Rich (SRCR) domain and a C-terminal trypsin-like serine peptidase (SP) 54 domain with a canonical Ser441-His296-Asp345 catalytic triad (Fig. 1a and e) ${ }^{7,8}$. As TMPRSS2 is 55 synthesized as a single-chain proenzyme, or zymogen, it requires cleavage at a conserved Arg25556 Ile256 peptide bond within its SRQSR255\IVGGE activation motif (cleavage site denoted with 57 an arrow) to achieve full maturation of its enzymatic activity ${ }^{8,9}$. We achieved this in high yield by 58 replacing SRQSR255 $\downarrow$ with an enteropeptidase-cleavable DDDDK255 $\downarrow$ sequence to prohibit auto59 activation, allowing purification of a secreted form of the full TMPRSS2 ectodomain zymogen 60 from insect cells, analogous to a strategy used for the TTSP, matriptase (Methods) ${ }^{10}$. Subsequent 61 proteolytic activation with recombinant enteropeptidase afforded highly active, homogenous 62 TMPRSS2 to milligram yields and was accordingly named directed activation strategy TMPRSS2 63 (dasTMPRSS2; Fig. 1b; Extended Data Fig. 1). We determined the X-ray crystal structure of 64 dasTMPRSS2 refined to $1.95 \AA$ resolution after acylation of the catalytic Ser441 residue with 65 nafamostat, a broad-spectrum synthetic serine protease inhibitor ${ }^{11}$. We obtained clear electron 66 density for residues 149-491 spanning the SRCR and SP domains but not residues 109-148 67 containing the flexible LDLR-A domain responsible for linking the protease to the plasma 68 membrane (Fig. 1c). The engineered DDDDK255 activation motif was not resolved in the structure 69 but rather terminated in an unstructured loop, consistent with matured TMPRSS13 (6KD5) and 70 hepsin $(1 \mathrm{Z8G})$ structures containing their native activation motifs ${ }^{12,13}$. The newly exposed $\mathrm{N}$ 71 terminal Ile256 of the SP domain formed a salt bridge with the side chain of Asp440, confirming 72 full maturation of the activation pocket, and taken together with the Cys244-Cys365 interdomain 73 disulfide, confirms that this structure represents the bioactive, stabilized form of the protease (Fig. 74 1d-e). 


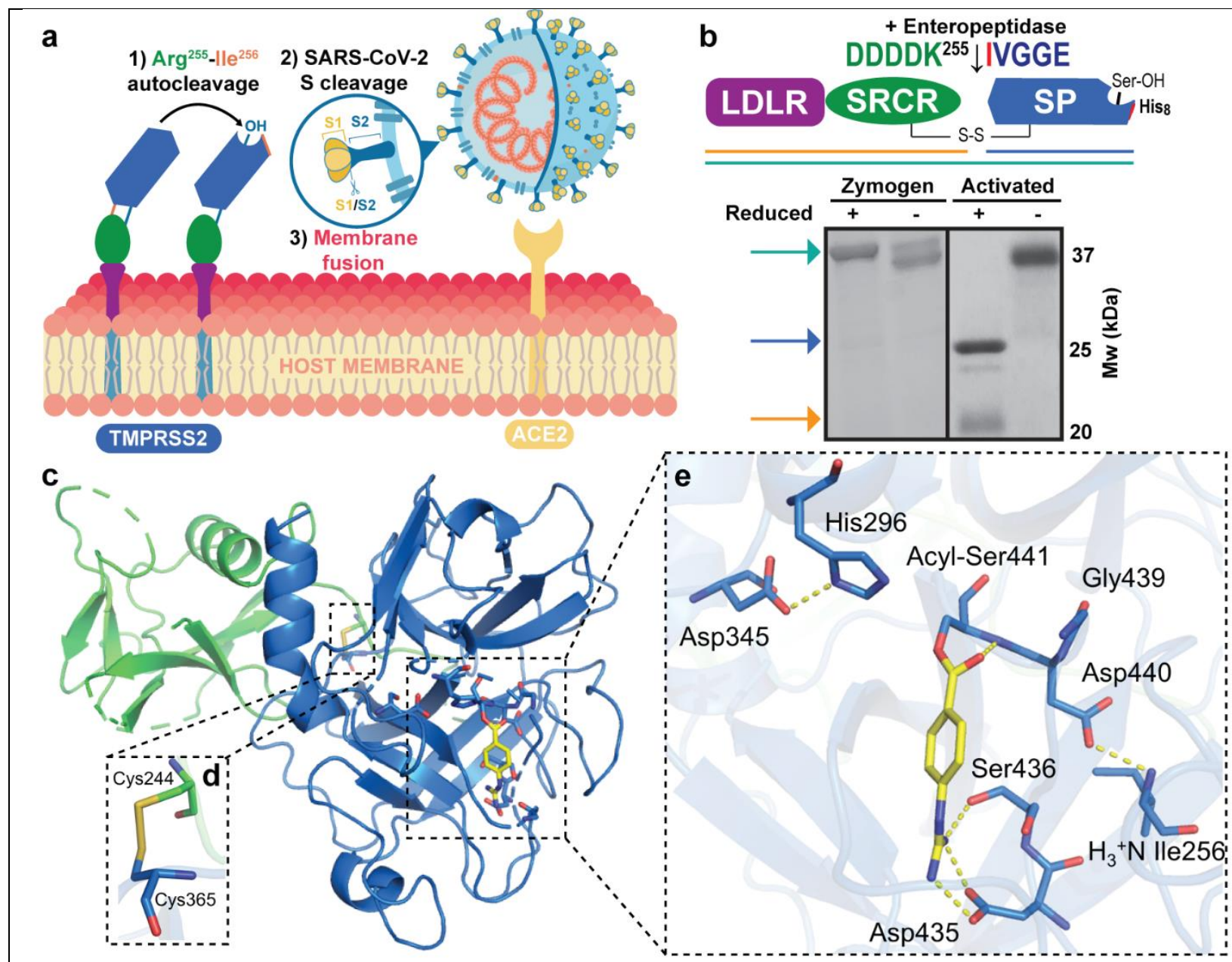

Figure 1. Engineered activation and structural characterization of stabilized TMPRSS2 ectodomain. a Full-length, membrane bound TMPRSS2 zymogen undergoes autocleavage activation at the Arg255-Ile256 peptide bond and the matured enzyme proteolytically processes SARS-CoV-2 Spike protein docked to the ACE2 receptor to drive viral membrane fusion. b Engineered recombinant TMPRSS2 ectodomain containing the low-density lipoprotein receptor type-A (LDLR) domain, a Class A Scavenger Receptor Cysteine-Rich (SRCR) domain and a C-terminal trypsin-like serine peptidase (SP) domain, features an enteropeptidase-cleavable DDDDK $^{255}$ substitution to facilitate controlled zymogen activation. The non-catalytic (LDLR+SRCR) and catalytic (SP) chains are tethered by a disulfide bond and the activation status can be interrogated by SDS-PAGE under non-reducing and reducing (5\% $\beta$ mercaptoethanol) conditions. $\mathbf{c}$ X-ray crystal structure of activated TMPRSS2 ectodomain pre-treated with nafamostat (yellow sticks). d The interdomain disulfide pair (Cys244-Cys365) maintains covalent attachment of the SRCR and SP domains. e Close-up view of the SP catalytic triad residues (His296, Asp345 and Ser441) and the post-activation Asp440:Ile256 salt bridge showing complete maturation of the protease. Nafamostat treatment results in phenylguanidino acylation of Ser441. Polar contacts are shown as yellow dashed lines.

\section{TMPRSS2 has a unique and accommodating substrate binding cleft}

The TMPRSS2 SP domain is highly conserved with all TTSPs and conforms to the canonical chymotrypsin/trypsin fold with two six-stranded beta barrels converging to a central active site cleft harboring the catalytic triad (Fig. 1c) ${ }^{14}$. Divergent protein substrate specificity of these closely related proteases is conferred through highly variable, surface-exposed loops, denoted Loop A-E 
and Loops 1-3 (Extended Data Fig. 2) ${ }^{14}$. Unique subsites formed on the face of the SP domain, S4S3-S2-S1-S1'-S2'-S3'-S4' recognize substrate P4-P3-P2-P1 $\downarrow$ P1'-P2'-P3'-P4' amino acid positions spanning the scissile bond (Fig 2a; Extended Data Fig 3a). To rationally assign these subsites for TMPRSS2, we superposed the peptide-bound hepsin and TMPRSS13 SP domains ( $40.1 \%$ and $41.4 \%$ sequence identity of their SP domains, respectively) belonging to the same hepsin/TMPRSS subfamily as TMPRSS2. The S1 position of TMPRSS2 is occupied by the phenylguanidino moiety of nafamostat, forming salt bridges with the highly conserved Asp435, Ser436, and Gly464 residues in the same binding mode as the guanidino of P1 Arg residues observed in hepsin and TMPRSS13 (Fig. 1e; Fig. 2a; Extended Data Fig. 3a). The TMPRSS2 S2 subsite has a distinguishing Lys342 residue that likely confers a preference for small and/or electronegative P2 substrates, similar to the S2 Lys in enteropeptidase which prefers P2 Asp residues ${ }^{15}$. The S3 and S4 subsites appear open to accommodate various $\mathrm{P} 3$ and $\mathrm{P} 4$ amino acids and may make favorable receptor contacts with the respective Gln438 and Thr341 positions (Fig. 2a, Extended Data Fig. 3a). On the N-terminal side of the scissile bond, the buried S1' site appears to accept small, hydrophobic P1' residues. Overall, the TMPRSS2 active site appears capable of binding various substrate sequences with the strictest preference for the $\mathrm{P} 1$ and $\mathrm{P} 2$ positions.

Among TTSPs, the SP domain of TMPRSS2 uniquely possesses 3 disulfides and a single unpaired cysteine residue, Cys379 (Extended Data Fig. 2b-d). In all other TTSPs this position forms a disulfide bond with an additional Cys at the equivalent of Thr447, or both cysteines are absent. This unpaired cysteine is conserved in feline, bovine, mouse, and rat TMPRSS2 orthologs (Extended Data Fig. 2e). Furthermore, the unpaired Cys 379 is bordered by an expansive $360 \AA^{2}$ patch of exposed hydrophobic surface area in our structure that may serve as an interaction hub for TMPRSS2 binding partners (Extended Data Fig. 2b).

\section{The SRCR domain confers additional diversity for molecular recognition.}

The SRCR domain is found enriched in proteins expressed at the surface of immune cells as well as in secreted proteins, and are thought to participate in protein-protein interactions and substrate recognition ${ }^{16}$. The Class A SRCR domain of TMPRSS2 is located on the backside of the SP domain away from the active site and is structurally similar to that of TMPRSS13 despite sharing only 19\% sequence identity (Fig. 2b). These two SRCR domains adopt a compact, globular fold with similar orientations relative to their SP domains (Fig. 2b,c). The SRCR of hepsin (7.5\% sequence identity) diverges significantly from TMPRSS $2 / 13$ with three intra-domain disulfides and a tighter SRCR:SP association dominated by complementary electrostatic patches and buried surface area (Fig. 2c). These conformational differences may play a role in the orientation of the SP domain relative to the plasma membrane as well as modulate activity through recognition or recruitment of partner proteins. 




Figure 2. Divergent surface properties of TMPRSS2 inform putative substrate preferences and relative domain organization. a The subsites of TMPRSS2 (blue) superimposed on the corresponding residues of Hepsin (magenta, PDB: 1Z8G) and TMPRSS13 (orange, PDB: 6KD5). The S1 subsite occupied by the phenylguanidino acyl group has well conserved Ser441, Asp435, and Gly464 residues, whereas discriminatory residues in S2 (Lys342) and S4 (Thr341) are not occupied. b Ribbon representation of the superimposed SRCR domains of TMPRSS2 (blue), Hepsin (magenta, PDB: 1Z8G) and TMPRSS13 (orange, PDB: 6KD5). $\mathbf{c}$ Relative orientation of the SRCR (green) and serine protease domains (blue) of TMPRSS2, Hepsin (PDB: 1Z8G), and TMPRSS13 (PDB: 6KD5). The LDLR domain of TMPRSS13 is shown in magenta with bound calcium in orange.

\section{TMPRSS2 displays robust in vitro peptidase activity}

124 To evaluate TMPRSS2 inhibitors and provide groundwork for future structure activity relationship 125 (SAR) studies, we established in vitro proteolytic activity and inhibition assays. The generic TTSP 
fluorogenic peptide substrate Boc-Gln-Ala-Arg-7-aminomethylcoumarine (AMC) was rapidly cleaved by dasTMPRSS2, C-terminal to Arg, thereby releasing AMC product and enabling initial reaction velocities $\left(V_{o}\right)$ measurement within 60 seconds of enzyme addition (Fig. 3a). In Assay Buffer, dasTMPRSS2 had a $K_{m}$ of $(200 \pm 80) \mu \mathrm{M}, V_{\text {max }}$ of $(0.7 \pm 0.2) \mathrm{nmol} \mathrm{min}^{-1}, k_{\text {cat }}$ of $(18 \pm 4) \mathrm{s}^{-1}$,

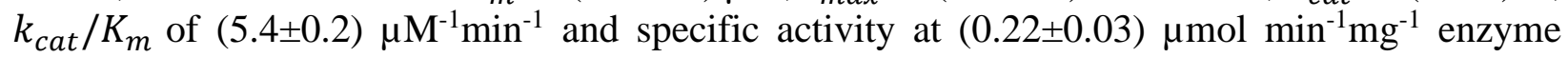
purified to apparent homogeneity (Fig. 3b). To our knowledge, this level of activity has not been achieved with any previously described recombinant TMPRSS2 enzyme ${ }^{17-20}$, and enzyme activity was unaffected by the presence of $\mathrm{Ca}^{2+}, \mathrm{NaCl}$ concentrations ranging 75-250 $\mathrm{mM}$, EDTA, and tolerant of $2 \%(\mathrm{v} / \mathrm{v})$ DMSO (Extended Data Fig. 4c) that is encouraging for use in high throughput inhibitor screening campaigns.

\section{TMPRSS2 efficiently cleaves the SARS-CoV-2 S protein at the S1/S2 site in vitro}

Cells expressing TMPRSS2 have been shown to efficiently cleave the SARS-CoV-1 S protein at S1/S2 (SLLR667 $\downarrow$ ) and multiple peripheral sites to induce the necessary conformational changes leading to virus-host fusion at the plasma membrane ${ }^{21,22}$ (Fig. 1a; Extended Data Fig. 5a). This extensive TMPRSS2 processing has also been linked to periplasmic shedding of the S1 fragment to act as an immune decoy in vivo ${ }^{22}$. For SARS-CoV-2, an acquired multibasic RRAR685 $\downarrow$ S1/S2 cleavage sequence was hypothesized to confer preferential cleavage by intracellular furin protease $^{23}$, and was corroborated by studies showing that multibasic, peptidomimetic furin inhibitors prevented S1/S2 cleavage and attenuate infection in cellular models ${ }^{24}$. Further studies showed that these inhibitors are promiscuous and disable multiple surface-expressed proteases that process multibasic substrates in addition to furin, and more selective furin inhibitors cannot fully abrogate $\mathrm{S}$ activation ${ }^{25}$. Furthermore, furin-deficient cells can still generate $\mathrm{S} 1 / \mathrm{S} 2$ cleaved virus, and propagation of SARS-CoV-2 in TMPRSS2-deficient cell lines results in a loss of the multibasic S1/S2 site ${ }^{26}$, attenuating viral infectivity towards TMPRSS2+ cells. We sought to characterize TMPRSS2's proteolytic activity towards S1/S2 by incubating recombinant furin and/or dasTMPRSS2 with stabilized SARS-CoV-2 S protein ectodomain with S1/S2 knocked out (RRAR $^{685}{ }_{-}$GSAS $^{685}$; HexaPro construct) or with S1/S2 intact (denoted HexaFurin; Extended Data Fig. 5a; Fig. 3c). As expected from previous studies using recombinant, S1/S2 intact S protein, HexaFurin sustained partial S1/S2 cleavage during production in HEK293 cells due to endogenously expressed furin ${ }^{27}$ (Fig. 3c). Recombinant furin treatment converted the remaining intact HexaFurin to S1 and S2 band fragments with incubation over 16 hours, but was unable to cleave HexaPro (Fig. 3c; Extended Data Fig. 5b).

In contrast, using both the HexaFurin and HexaPro constructs, we observed that dasTMPRSS2 could cleave the $\mathrm{S}$ protein at 3 distinct sites with variable efficiency (Fig. 3d-e). HexaFurin was cleaved to only the S1 and S2 fragments within 5 minutes of dasTMPRSS2 addition (Fig. 3d), demonstrating the S1/S2 site was best recognized by TMPRSS2 across a minimal incubation. HexaPro, lacking S1/S2, was cleaved across 30 min to generate a larger $150 \mathrm{kDa}$ band, denoted fragment $\mathrm{X}$, and $80 \mathrm{kDa}$ fragment $\mathrm{Y}$ when analyzed under non-reducing conditions (Fig. 3e). Reducing conditions revealed an additional cleavage site hidden within fragment $X$ that is spanned by two cysteine residues participating in a disulfide bond, splitting fragment $\mathrm{X}$ into $120 \mathrm{kDa}$ fragment X'a and $35 \mathrm{kDa}$ fragment X'b. Exhaustive HexaPro treatment (120 min) completely converted fragment $X$ into $X^{\prime} a$ and $X^{\prime} b$ (Extended Data Fig. 6d). 

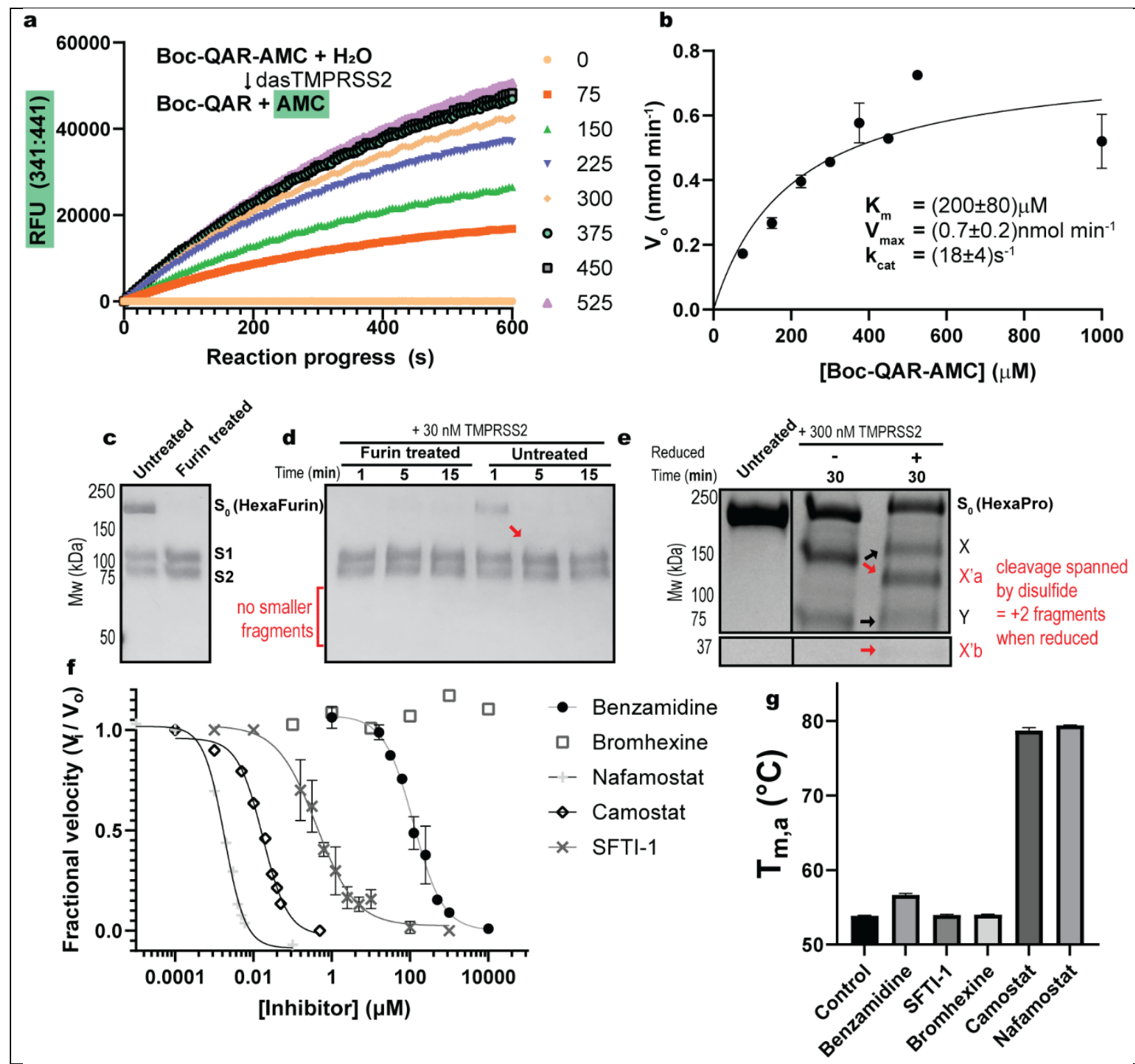

Figure 3. dasTMPRSS2 displays robust proteolytic activity towards peptide and SARS-CoV-2 S protein substrates. a The generic Boc-Gln-Ala-Arg-AMC fluorogenic peptide substrate is efficiently cleaved by dasTMPRSS2. Each progress curve was performed in quadruplet. b Michaelis-Menten plot of initial reaction velocities for kinetic parameter estimation after curve fitting in GraphPad. c S1/S2 intact $\mathrm{S}$ protein ectodomain (HexaFurin; $\mathrm{S}_{0}$ ) is partially cut at the $\mathrm{S} 1 / \mathrm{S} 2$ site to produce $\mathrm{S} 1$ and $\mathrm{S} 2$ fragments and can be quantitatively converted by recombinant furin treatment over 16 hours. $\mathbf{d}$ The addition of $30 \mathrm{nM}$ TMPRSS2 to furin treated HexaFurin produces no additional bands, but furin untreated HexaFurin is exhaustively cleaved at S1/S2 within 5 minutes. e dasTMPRSS2 cleaves HexaPro at two additional sites peripheral to S1/S2, with the first cleavage producing $\mathrm{X}$ and $\mathrm{Y}$ band fragments under non-reducing SDS-PAGE conditions. Reducing conditions reveal band fragments $X^{\prime}$ a and $X^{\prime}$ 'b derived from fragment $\mathrm{X}$. $\mathbf{f}$ dasTMPRSS2 peptidase activity is blocked with varying potencies by clinical protease inhibitors, with no inhibition seen for bromhexine. All data are shown as mean \pm s.e.m., $n=3$ biological replicates $\mathbf{g}$ Apparent melting temperatures (as determined by differential scanning fluorimetry) are increased for benzamidine, camostat, and nafamostat at $1 \mu \mathrm{M}$ concentration but are not increased by SFTI-1 or bromhexine. Samples were in triplicate. 
171 To visualize all of these cleavage sites simultaneously, we treated HexaFurin 30 min with dasTMPRSS2 and compared SDS-PAGE banding to a western blot using an antibody directed towards the $\mathrm{S}$ protein receptor binding domain (RBD; Extended Data Fig. 5c). At least 7 bands were observed on reducing SDS-PAGE and the western shows that both the $\mathrm{S} 1$ fragment as well as an S1-derived $50 \mathrm{kDa}$ fragment contain the RBD. The banding patterns observed (S1/S2, X/Y, and $X^{\prime} a / X^{\prime} b$ cleavages) are consistent with western blot studies monitoring SARS-CoV-1 S protein processing by TMPRSS 2 that enables shedding of the $\mathrm{S} 1$ fragment $^{22}$ to act as an immune decoy.

\section{Nafamostat rapidly acylates TMPRSS2 and slowly hydrolyzes}

Nafamostat and camostat are serine protease inhibitors under investigation as anti-TMPRSS2 COVID-19 therapeutics (Clinical Trial.gov identifiers NCT04583592, NCT04625114). Both are reactive esters that form the same slowly-reversible phenylguanidino covalent complex (evidenced in the enteropeptidase-camostat structure; PDB: 6ZOV) with the catalytic serine residue of trypsinlike serine proteases. Nafamostat and camostat dramatically increased the apparent melting temperature $\left(T_{M, a}\right)$ of dasTMPRSS2 by $(25.5 \pm 0.1)$ and $(24.8 \pm 0.3){ }^{\circ} \mathrm{C}$, respectively, as measured by Differential Scanning Fluorimetry (DSF) ${ }^{28}$ (Fig. 3g) and was a key stabilizing feature to enable protein crystallization (Methods). Nafamostat demonstrated enhanced potency over camostat with $\mathrm{IC}_{50}$ values of $(1.7 \pm 0.2)$ and $(17 \pm 4) \mathrm{nM}$, respectively, with 5 min assay pre-incubation (Fig. 3f). However, IC 50 values were time-dependent and required further kinetic interrogation to assess their divergent potencies (Extended Data Fig. 6b-c). Nafamostat was 40-fold more potent than camostat with respective $k_{\text {inact }} / K_{\mathrm{i}}$ values of $(0.024 \pm 0.006)$ and $(0.00059 \pm 0.00003) \mathrm{s}^{-1} \mathrm{nM}^{-1}$. These results emphasize that single timepoint $\mathrm{IC}_{50}$ values are insufficient for evaluating mechanism-based, covalent inhibitors of this highly active protease in SAR studies. As previously identified for matriptase, the nafamostat leaving group, 6-amidino-2-napthol, fluoresces and can be used as a sensitive burst titrant to calculate the concentration of active protease (Extended Data Fig. 4e-f; Methods $)^{19}$. The half-life of the phenylguanidino acyl-enzyme complex was $(14.7 \pm 0.4)$ hours as measured by the gradual rescue of dasTMPRSS2 peptidase activity after stoichiometric acylation with nafamostat (Extended Data Fig. 3e-f). Impressively, stoichiometric amounts of nafamostat completely blocked dasTMPRSS2-mediated HexaPro activation over 2 hours (Extended Data Fig. 6d) and are consistent with this drug's ability to potently block SARS-CoV2 pseudovirus entry to TMPRSS $2+$ Calu- $3^{29}$ and Caco- $2^{5,20}$ lung cells.

Non-covalent trypsin-like serine protease inhibitors benzamidine and sunflower trypsin inhibitor1 (SFTI-I) were less potent with respective IC50 values of $(120 \pm 20) \mu \mathrm{M}$ and $(0.4 \pm 0.2) \mu \mathrm{M}$ (Fig. $3 \mathrm{f}$ ), and $K_{i}$ values of $(80 \pm 10) \mu \mathrm{M}$ and $(0.4 \pm 0.2) \mu \mathrm{M}$ (Extended Data Fig. 7a-b). 6-amidino-2napthol also disabled dasTMPRSS2 activity with an $\mathrm{IC}_{50}$ of $(1.6 \pm 0.5) \mu \mathrm{M}$ and $K_{i}$ of $(1.1 \pm 0.3) \mu \mathrm{M}$ (Extended Data Fig. 7a). Bromhexine hydrochloride, another agent under investigation for antiTMPRSS2 COVID-19 therapy ${ }^{30,31}$, showed no inhibition in either the peptidase or HexaPro cleavage assay formats (Extended Data Fig. 7c-d), corroborating reports of its ineffectiveness in blocking SARS-CoV-2 pseudovirus entry ${ }^{32}$ and further underscores the need for novel, selective TMPRSS2 inhibitors.

\section{Future prospects}

We have produced and characterized a source of TMPRSS2 enzyme that will enable rapid inhibitor development as antivirals and thorough molecular interrogation of coronavirus and influenza virus 
activation. Although nafamostat potently neutralizes TMPRSS2 activity, it is non-selective and disables trypsin-like serine proteases involved in coagulation such as plasmin, FXa, and FXIIa, as well as other TTSPs through its generic arginine-like engagement with the S1 subsite ${ }^{19,33,34}$. Furthermore, nafamostat requires continuous intravenous infusion to approach therapeutic concentrations for COVID-19 owing to its short biological half-life of 8 minutes (NCT04418128; NCT04473053). These features, although undesirable as a selective therapeutic, make nafamostat an extremely useful and sensitive reagent for in vitro kinetic characterization of trypsin-like proteases, and sufficiently stabilized our protease for crystallization and structural determination. Nevertheless, selective and biologically stable drugs for TMPRSS2 must be explored, and may be achieved through inhibitors engaging the more TMPRSS2-specific S2, S3, and S4 subsites identified in our crystal structure.

We observed no electron density for the LDLR-A domain of TMPRSS2, despite a similar construct design to that which afforded the TMPRSS13 crystal structure (PDB: 6KD5). The LDLR-A domain of TTSPs is responsible for tethering the protease to the plasma membrane and most TTSPs have a conserved ability to bind calcium. Interestingly, a key Asp residue in TMPRSS13 involved in calcium chelation is absent in human and other mammalian TMPRSS2 proteins, substituted instead with His or Gln residues (Extended Data Fig. 8). These data suggest that TMPRSS2 may have lost the ability to bind calcium at this site.

Our demonstration that TMPRSS2 can cleave the multibasic S1/S2 site of the S protein suggests that instead of conferring furin dependence, the virulent properties of this site may derive from promiscuous recognition and cleavage by airway-expressed TTSPs, which is supported by the demonstrated roles that TMPRSS4 ${ }^{35,36}$, TMPRSS11 $\mathrm{d}^{20,37,38}$, and TMPRSS13 ${ }^{20,37}$, which colocalize with ACE2 ${ }^{36}$, play in enabling SARS-CoV-2 infection across various tissues.

Our characterization of dasTMPRSS2 did not reveal an obvious mechanism by which the native, membrane-bound enzyme could be autoproteolytically processed peripheral to the activation motif and thereby shed as a soluble enzyme into the extracellular space. However, studies using TMPRSS2-specific antibodies have reported detection of a secreted enzyme product in prostate sera that is expected to play a functional role in pericellular activation ${ }^{39}$. Due to the disulfide-linked nature of activated TMPRSS2, former studies may have mischaracterized the catalytic subunit as a shed SP domain when it would instead resolve to the intact species under non-reducing conditions (Fig. 1b). Thus, a biochemical characterization of these secreted species is required to interpret their activation status and subunit organization, as an active, shed form of TMPRSS2 in the extracellular milieu would have profound pathobiological and therapeutic targeting implications.

\section{ACKNOWLEDGEMENTS}

We thank Jason McLellan for generously providing the SARS-CoV-2 S protein construct plasmids, Irene Chau for assistance with DSF, and Shih-Ting Tseng for preparation of Figure 1 graphic art. This work was supported by BC Leadership Chair in Functional Cancer Imaging to FB, the Canada Excellence Research Chair to SS, and a Mitacs Accelerate Internship to BF. This work is based upon research conducted at the Northeastern Collaborative Access Team beamlines, which are funded by the National Institute of General Medical Sciences from the National 
a NIH-ORIP HEI grant (S10OD021527). This research used resources of the Advanced Photon Source, a U.S. Department of Energy (DOE) Office of Science User Facility operated for the DOE Office of Science by Argonne National Laboratory under Contract No. DE-AC02-06CH11357. The Structural Genomics Consortium is a registered charity (no: 1097737) that receives funds from AbbVie, Bayer AG, Boehringer Ingelheim, Genentech, Genome Canada through Ontario Genomics Institute [OGI-196], the EU and EFPIA through the Innovative Medicines Initiative 2 Joint Undertaking [EUbOPEN grant 875510], Janssen, Merck KGaA (aka EMD in Canada and US), Pfizer, Takeda and the Wellcome Trust [106169/ZZ14/Z].

\title{
Author Information
}

These authors contributed equally: Bryan J. Fraser, Serap Beldar

\section{Author Affiliations}

Department of Molecular Oncology, British Columbia Cancer Research Institute, Vancouver, British Columbia, Canada

Bryan J. Fraser, Daniel Kwon, Ruiyan Tan, Ryan P. Wilson, François Bénard

\section{Department of Radiology, University of British Columbia, Vancouver, British Columbia, Canada}

Bryan J. Fraser, François Bénard

Structural Genomics Consortium, University of Toronto, Toronto, Ontario, Canada

Serap Beldar, Almagul Seitova, Ashley Hutchinson, Yanjun Li, Levon Halabelian, Cheryl H. Arrowsmith

Department of Biochemistry and Molecular Biology, University of British Columbia, Vancouver, British Columbia, Canada

Dhiraj Mannar, Karoline Leopold, Sriram Subramaniam

\section{Princess Margaret Cancer Centre, Toronto, Ontario, Canada}

Cheryl H. Arrowsmith

\author{
Department of Medical Biophysics, University of Toronto, Toronto, Ontario, Canada \\ Cheryl H. Arrowsmith
}

Author Contributions:

F.B., C.H.A., L.H., and S.S. provided project supervision; B.J.F., S.B., A.S., C.H.A., and F.B. conceived the project; B.J.F., S.B., A.S., C.H.A., and L.H. designed the experiments; Y.L. cloned TMPRSS2 protein constructs for expression and Y.L. and D.M cloned SARS-CoV-2 S protein constructs for expression; A.S. and A.H. produced TMPRSS2 protein in insect cells; A.S., A.H., D.M., K.L. produced SARS-CoV-2 S protein in HEK cells; B.J.F., S.B., A.S., A.H., D.M., and K.L. purified recombinant proteins; B.J.F., S.B., and L.H. crystallized TMPRSS2 and L.H., S.B., and B.J.F. collected diffraction data; L.H. solved the crystal structure; B.J.F., S.B., and L.H. performed bioinformatic and structural analyses; B.J.F., D.K., R.W., and R.T. performed 
synthesized, purified, and characterized SFTI-1 peptide; B.J.F., D.K., and S.B. managed inhibitor compound libraries; B.J.F., D.M., and R.T. performed gel-based S protein digestion assays; S.B. and B.J.F. performed DSF assays; B.J.F., S.B., L.H., R.T., and D.M. prepared figures; B.J.F., S.B., L.H., C.H.A., F.B., A.S., A.H., and Y.L. wrote the manuscript.

\section{Competing interests}

316

The authors declare no competing interests.

\section{Data Availability}

The coordinates and structure of the phenylguanidino TMPRSS2 acyl-enzyme complex have been deposited in the PDB with accession number 7MEQ on April 7, 2021, and released on April 21, 2021. Any other relevant data are available from the corresponding authors upon reasonable request.

\section{REFERENCES}

1. Matrosovich, T., Beyerle, M., Klenk, H., Garten, W. \& Matrosovich, M. Proteolytic Activation of Influenza Viruses by Serine Proteases TMPRSS2 and HAT from Human Airway Epithelium. 80, 9896-9898 (2006).

2. Böttcher, E. et al. Proteolytic Activation of Influenza Viruses by Serine Proteases TMPRSS2 and HAT from Human Airway Epithelium. J. Virol. 80, 9896-9898 (2006).

3. Limburg, H. et al. TMPRSS2 Is the Major Activating Protease of Influenza A Virus in Primary Human Airway Cells and Influenza B Virus in Human Type II Pneumocytes. $J$. Virol. 93, 1-22 (2019).

4. Matsuyama, S. et al. Efficient Activation of the Severe Acute Respiratory Syndrome Coronavirus Spike Protein by the Transmembrane Protease TMPRSS2. J. Virol. 84, 12658-12664 (2010).

5. Hoffmann, M. et al. SARS-CoV-2 Cell Entry Depends on ACE2 and TMPRSS2 and Is Blocked by a Clinically Proven Article SARS-CoV-2 Cell Entry Depends on ACE2 and TMPRSS2 and Is Blocked by a Clinically Proven Protease Inhibitor. 271-280 (2020) doi:10.1016/j.cell.2020.02.052.

6. Li, J., Zhan, P. \& Liu, X. Targeting the entry step of SARS-CoV-2: a promising therapeutic approach. Signal Transduct. Target. Ther. 5, 2019-2020 (2020).

7. Paoloni-giacobino, A., Chen, H., Peitsch, M. C., Rossier, C. \& Antonarakis, S. E. Cloning of the TMPRSS2 Gene, Which Encodes a Novel Serine Protease with Transmembrane, LDLRA, and SRCR Domains and Maps to 21q22 . 3. 320, 309-320 (1997).

8. Chen, Y. et al. TMPRSS2, a Serine Protease Expressed in the Prostate on the Apical Surface of Luminal Epithelial Cells and Released into Semen in Prostasomes, Is Misregulated in Prostate Cancer Cells. 176, 2986-2996 (2010).

9. Afar, D. E. H. et al. Catalytic Cleavage of the Androgen-regulated TMPRSS2 Protease Results in Its Secretion by Prostate and Prostate Cancer Epithelia. 1686-1692 (2001).

10. Yamasaki, Y., Satomi, S., Murai, N., Tsuzuki, S. \& Fushiki, T. Inhibition of membranetype serine protease $1 /$ matriptase by natural and synthetic protease inhibitors. J. Nutr. Sci. Vitaminol. (Tokyo). 49, 27-32 (2003).

11. Fujii, S. (Received April 21st, 1981). Biochim. Biophys. Acta 661, 342-345 (1981).

354 12. Klezovitch, O. et al. Hepsin promotes prostate cancer progression and metastasis. Cancer 
Cell 6, 185-195 (2004).

13. Ohno, A. et al. Crystal structure of inhibitor-bound human MSPL that can activate high pathogenic avian in fl uenza. Life Sci. Alliance 4, 1-11 (2021).

14. Perona, J. J. \& Craik, C. S. Evolutionary divergence of substrate specificity within the chymotrypsin-like serine protease fold. J. Biol. Chem. 272, 29987-29990 (1997).

15. Boulware, K. T. \& Daugherty, P. S. Protease specificity determination by using cellular libraries of peptide substrates ( CLiPS ). Proc Natl Acad Sci U S A . (2006) doi:10.1073/pnas.0511108103.

16. Yap, N. V. L., Whelan, F. J., Bowdish, D. M. E. \& Golding, G. B. The evolution of the scavenger receptor cysteine-rich domain of the. 6, 1-9 (2015).

17. Lucas, J. M. et al. The androgen-regulated protease TMPRSS2 activates a proteolytic cascade involving components of the tumor microenvironment and promotes prostate cancer metastasis. Cancer Discov. 4, 1310-1325 (2014).

18. Meyer, D., Sielaff, F., Hammami, M. \& Ottcher-friebertsh, E. B. Identification of the first synthetic inhibitors of the type II transmembrane serine protease TMPRSS2 suitable for inhibition of influenza virus activation. 343, 331-343 (2013).

19. Nimishakavi, S., Raymond, W. W., Gruenert, D. C. \& Caughey, G. H. Divergent inhibitor susceptibility among airway lumen-accessible tryptic proteases. PLoS One 10, 1-17 (2015).

20. Hoffmann, M. et al. Camostat mesylate inhibits SARS-CoV-2 activation by TMPRSS2related proteases and its metabolite GBPA exerts antiviral activity. EBioMedicine 65, (2021).

21. Reinke, L. M. et al. Different residues in the SARS-CoV spike protein determine cleavage and activation by the host cell protease TMPRSS2. PLoS One 12, 1-15 (2017).

22. Glowacka, I. et al. Evidence that TMPRSS2 Activates the Severe Acute Respiratory Syndrome Coronavirus Spike Protein for Membrane Fusion and Reduces Viral Control by the Humoral Immune Response. J. Virol. 85, 4122-4134 (2011).

23. Cells, L., Hoffmann, M. \& Kleine-weber, H. Short Article A Multibasic Cleavage Site in the Spike Protein of SARS-CoV-2 Is Essential for Infection of Human 1111 Short Article A Multibasic Cleavage Site in the Spike Protein of SARS-CoV-2 Is Essential for Infection of Human Lung Cells. 779-784 (2020) doi:10.1016/j.molcel.2020.04.022.

24. Bestle, D. et al. TMPRSS2 and furin are both essential for proteolytic activation of SARSCoV-2 in human airway cells. 3, 1-14 (2020).

25. Tang, T. et al. Proteolytic Activation of SARS-CoV-2 Spike at the S1/S2 Boundary: Potential Role of Proteases beyond Furin. ACS Infect. Dis. 7, 264-272 (2021).

26. Id, M. S., Uemura, K., Sato, A. \& Toba, S. SARS-CoV-2 variants with mutations at the S1 / S2 cleavage site are generated in vitro during propagation in TMPRSS2-deficient cells. 1-17 (2021) doi:10.1371/journal.ppat.1009233.

27. Gobeil, S. M. et al. Article D614G Mutation Alters SARS-CoV-2 Spike Conformation and Enhances Protease Cleavage at the S1 / S2 Junction 11 1l D614G Mutation Alters SARS-CoV-2 Spike Conformation and Enhances Protease Cleavage at the S1 / S2 Junction. CellReports 34, 108630 (2021).

28. Niesen, F. H., Berglund, H. \& Vedadi, M. The use of differential scanning fluorimetry to detect ligand interactions that promote protein stability. Nat. Protoc. 2, 2212-2221 (2007).

29. Hoffmann, M., Schroeder, S., Kleine-weber, H., Müller, M. A. \& Drosten, C. Nafamostat Mesylate Blocks Activation of SARS-CoV-2 : New Treatment Option for COVID-19. 
Antimicrob Agents Chemother 64, 19-21 (2020).

30. Li, T. et al. Bromhexine Hydrochloride Tablets for the Treatment of Moderate COVID-

404 19 : An Open-Label Randomized Controlled Pilot Study. Clin. Transl. Sci. 13, 1096-1102 (2020).

407

31. Wang, Y. et al. Evaluating the efficacy and safety of bromhexine hydrochloride tablets in treating pediatric COVID-19. Medicine (Baltimore). 99, 0-4 (2020).

32. Shrimp, J. H. et al. An Enzymatic TMPRSS2 Assay for Assessment of Clinical Candidates and Discovery of Inhibitors as Potential Treatment of COVID-19. (2020).

33. Hitomi, Y, Ikari, N, Fujii, S. Inhibitory Effect of a New Synthetic Protease Inhibitor (FUT-175) on the Coagulation System. Haeomstasis 15, 164-168 (1985).

34. Muto, S., Imai, M. \& Asano, Y. Mechanisms of hyperkalemia caused by nafamostat mesilate. Gen. Pharmacol. 26, 1627-1632 (1995).

35. Zang, R. et al. TMPRSS2 and TMPRSS4 mediate SARS-CoV-2 infection of human small intestinal enterocytes. bioRxiv 3582, 1-14 (2020).

36. Wruck, W. \& Adjaye, J. SARS-CoV-2 receptor ACE2 is co-expressed with genes related to transmembrane serine proteases, viral entry, immunity and cellular stress. Sci. Rep. 10, 1-14 (2020).

37. Kishimoto, M. et al. Tmprss11d and tmprss13 activate the sars-cov-2 spike protein. Viruses 13, (2021).

38. $\mathrm{Ph}, \mathrm{D}$. Intracellular autoactivation of TMPRSS11A, an airway epithelial transmembrane serine protease. (2020) doi:10.1074/jbc.RA120.014525.

39. Kesic, M. J., Meyer, M., Bauer, R. \& Jaspers, I. Exposure to ozone modulates human airway protease/antiprotease balance contributing to increased influenza a infection. PLoS One 7, 1-12 (2012). 


\section{METHODS}

\section{Construct design and cloning}

A construct encoding residues 109-492 comprising soluble TMPRSS2 ectodomain was amplified by two PCR fragments (Addgene plasmid\# 53887) and subcloned into the pFHMSP-LIC C donor plasmid by LIC method. The final construct contained a $\mathrm{N}$-terminal honeybee melittin signal sequence peptide and C-terminal Hiss-tag (Fig.1b). Mutations targeting the activation sequence SSQSR255 $\downarrow$ IVGGE (arrow indicates the cleavage site) were implemented to replace the SRQSR255 residues with an enteropeptidase-cleavable DDDDK255 graft with two sets of primer pairs (Extended Data Table 1) generating mutations for S251D/R252D/Q253D/S254D/R255K. Plasmid transfer vector containing the TMPRSS2 gene was transformed into Escherichia coli DH10Bac cells (Thermo Fisher; Cat\# 10361012) to generate recombinant viral bacmid DNA. Sf9 cells were transfected with Bacmid DNA using JetPrime transfection reagents (PolyPlus Transfection Inc.; Cat\# 114-01) according to the manufacturer's instructions, and recombinant baculovirus particles were obtained and amplified from P1 to P2 viral stocks. Recombinant P2 viruses were used to generate suspension culture of baculovirus infected insect cells (SCBIIC) for scaled-up production of TMPRSS2.

The SARS-CoV-2 Spike ectodomain HexaPro construct was a gift from J. McLellan ${ }^{1}$, and the $\mathrm{S} 1 / \mathrm{S} 2$ site was restored $\left(\mathrm{GSAS}^{685}{ }_{-}>\mathrm{RRAR}\right)$ through site-directed mutagenesis with primers in Extended Data Table 1 (HexaFurin construct).

\section{Baculovirus mediated dasTMPRSS2 protein production in Sf9 insect cells}

Sf9 cells were grown in I-Max Insect Medium (Wisent Biocenter; Cat\# 301-045-LL) to a density of $4 \times 10^{6}$ cells $/ \mathrm{mL}$ and infected with $20 \mathrm{~mL} / \mathrm{L}$ of suspension culture of baculovirus infected insect cells prior to incubation on an orbital shaker $\left(145 \mathrm{rpm}, 26^{\circ} \mathrm{C}\right)$.

\section{dasTMPRSS2 protein purification}

Cell culture medium containing the final secreted protein product AA-[TMPRSS2(109-492)]EFVEHHHHHHH was collected by centrifugation $\left(20 \mathrm{~min}, 10{ }^{\circ} \mathrm{C}, 6,000 \mathrm{x} \mathrm{g}\right) 4-5$ days postinfection when cell viability dropped to $55-60 \%$. Media was adjusted to $\mathrm{pH} 7.4$ by addition of concentrated PBS stock, then supplemented with $15 \mathrm{~mL} / \mathrm{L}$ settled Ni-NTA resin (Qiagen) at a scale of $12 \mathrm{~L}$. Three batch Ni-NTA purifications were used to capture protein, with each round requiring shaking in $2 \mathrm{~L}$ flasks for 2 hours at $16^{\circ} \mathrm{C}(110 \mathrm{rpm})$, harvesting by centrifugation $(5 \mathrm{~min} 1,000 \mathrm{x}$ $\mathrm{g})$, then transferred to a gravity flow column. Beads were washed with 3 column volumes (CVs) ice-cold PBS prior to elution with PBS supplemented with $500 \mathrm{mM}$ imidazole. Elution samples were concentrated to $4.5 \mathrm{mg} / \mathrm{mL}$ using $30 \mathrm{kDa}$ MWCO Amicon filters and overnight zymogen activation was achieved by dialyzing protein 1:1000 against Assay Buffer (25 mM Tris pH 8.0, 75 $\mathrm{mM} \mathrm{NaCl}, 2 \mathrm{mM} \mathrm{CaCl}$ ) at room temperature in the presence of recombinant enteropeptidase (NEB) at $13 \mathrm{U}$ enzyme per mg TMPRSS2 zymogen. The following day, activated samples were exchanged to SEC buffer (50 mM Tris $\mathrm{pH} 7.5,250 \mathrm{mM} \mathrm{NaCl}$ ), spun down at 17,000 x g, then loaded to a Superdex 75 gel filtration column. Fractions spanning the dominant peak eluting at 80 $\mathrm{mL}$ (Extended Data Fig. 1) were evaluated for appropriate banding on reducing SDS-PAGE prior to pooling and concentrating. For dasTMPRSS2 enzyme samples, $2 \mu \mathrm{L}$ aliquots of 10,000x enzyme assay stocks $(32 \mu \mathrm{M})$ were prepared by concentrating protein to $1.6 \mathrm{mg} / \mathrm{mL}$ in Enzyme Buffer (50 mM Tris $\mathrm{pH} 7.5,250 \mathrm{mM} \mathrm{NaCl}, 25 \%$ glycerol), then flash-frozen in liquid nitrogen 
and stored at $-80{ }^{\circ} \mathrm{C}$ until thawed immediately prior to use for each enzyme assay in order to minimize autoproteolysis and maintain reproducible enzyme concentrations.

\section{HexaPro and HexaFurin production and purification} plasmid encoding HexaPro/Furin using FectoPro transfection reagent (Polyplus-transfection ${ }^{\circledR}$ SA, Cat. \#116-010) with $5 \mathrm{mM}$ sodium butyrate being added at the time of transfection (Sigma, Cat. \# 303410). After 4-5 days post-transfection time cells culture was harvested, supernatant cleared by centrifugation, and the $\mathrm{pH}$ was adjusted by adding $10 \mathrm{x}$ Buffer $(50 \mathrm{mM}$ Tris $\mathrm{pH} 8.0,150 \mathrm{mM} \mathrm{NaCl})$. Secreted protein was captured by two round of batch absorption (BA) with $4 \mathrm{~mL} / \mathrm{L}$ of preequilibrated Ni Sepharose beads (GE Healthcare, Cat \#17-5318-01). The bound beads were transferred to gravity flow column and sequentially washed with $30 \mathrm{CVs}$ Wash Buffer (50 mM HEPES 7.5, $300 \mathrm{mM} \mathrm{NaCl}, 5 \%$ glycerol), followed by Wash Buffer supplemented with $25 \mathrm{mM}$ imidazole. Protein was eluted in Elution Buffer (Wash buffer with $250 \mathrm{mM}$ imidazole) and concentrated using Amicon ${ }^{\mathrm{TM}}$ Ultra Centrifugal Filter Units, $15 \mathrm{~mL}, 100 \mathrm{kDa}$ (Millipore Sigma ${ }^{\mathrm{TM}}$ Cat\# UFC910024) prior to size-exclusion chromatography purification using a Superose 6 Increase 10/300 GL (GE Healthcare Cat \# 29-0915-96), in a buffer composed of $20 \mathrm{mM}$ HEPES pH 7.5, $200 \mathrm{mM} \mathrm{NaCl}$.

\section{Protein crystallization and structural determination}

After size-exclusion purification of activated dasTMPRSS2, samples were pooled and concentrated to $2 \mathrm{mg} / \mathrm{mL}$. Protein was treated with 3:1 nafamostat:dasTMPRSS2 for 10 minutes at room temperature and exchanged into Assay Buffer supplemented with 3:1 nafamostat using 4 spin cycles in $30 \mathrm{kDa}$ Amicon MWCO filters $\left(14,000 \mathrm{rpm}, 15 \mathrm{~min}, 4^{\circ} \mathrm{C}\right)$ to remove low $\mathrm{Mw}$ autolytic fragments from the $42 \mathrm{kDa}$ enzyme (Extended Data Fig. 1b). Acylated enzyme was then concentrated to $8 \mathrm{mg} / \mathrm{mL}$ and centrifuged $\left(14,000 \mathrm{rpm}, 10 \mathrm{~min}, 4^{\circ} \mathrm{C}\right)$ prior to automated screening at $18{ }^{\circ} \mathrm{C}$ in 96-well Intelliplates (Art Robin) using the Phoenix protein crystallization dispenser (Art Robbins). Protein was dispensed as $0.3 \mu \mathrm{L}$ sitting drops and mixed 1:1 with precipitant. The RedWing and SGC precipitant screens were tested and amorphous, non-diffracting crystals were consistently produced when grown over 30\% Jeffamine ED-2001 (Hampton Research) with 100 $\mathrm{mM}$ HEPES $\mathrm{pH}$ 7.0. To acquire a diffraction quality crystal, acylated dasTMPRSS2 was treated with $50 \mathrm{U}$ PNGase $\mathrm{F}$ (NEB; $37^{\circ} \mathrm{C}$ for $45 \mathrm{~min}$ ) to trim $\mathrm{N}$-glycan branches, then centrifuged $(14,000$ $\mathrm{rpm}, 4^{\circ} \mathrm{C}, 10 \mathrm{~min}$ ) prior to setting $2 \mu \mathrm{L}$ hanging drops with 1:1 protein: precipitant and grown for 10 days. Crystals were then cryo-protected using reservoir solution supplemented with $\sim 5 \%(\mathrm{v} / \mathrm{v})$ ethylene glycol, and cryo-cooled in liquid nitrogen. X-ray diffraction data were collected on the beamline 24-ID-E at the Advanced Photon Source (APS). Data were processed with XDS ${ }^{2}$. Initial phases were obtained by molecular replacement in Phaser $\mathrm{MR}^{3}$, using (PDB: 1Z8G) as a starting model. Model building was performed in $\mathrm{COOT}^{4}$ and refined with Buster ${ }^{5}$. Structure validation was performed in Molprobity ${ }^{6}$. Data collection and refinement statistics are summarized in Extended Data Table 2.

533

\section{Gel electrophoresis and western blotting}

SDS-PAGE was carried out with $15 \mu \mathrm{L}$ Mini-Protean (BioRad) or $60 \mu \mathrm{L}$ Novex Wedgewell (Invitrogen) 4-20\% Tris-Glycine gels for 30 min under constant voltage at 200V. Protein samples were mixed with $4 x$ Laemelli buffer (BioRad) and subjected to differential reducing $( \pm 5 \mathrm{mM} \beta$ mercaptoethanol; Gibco), then boiling at $95^{\circ} \mathrm{C}$ for $5 \mathrm{~min}$ in order to probe the covalent nature of 
protein complexes and subunits. The Precision Plus Protein marker (BioRad) was used as a standard. For SARS-CoV-2 RBD western blotting, SDS-PAGE was carried out as described, followed by wet transfer in Transfer Buffer (25 mM Tris pH 8.3, $192 \mathrm{mM}$ glycine, 20\% MeOH (v/v)) to PVDF membrane $\left(80 \mathrm{~V}, 53 \mathrm{~min}, 4^{\circ} \mathrm{C}\right)$. Membranes were incubated in Blocking Buffer $(5 \%$ skim milk in TBST) for $1 \mathrm{hr}$ at room temperature, washed $5 \mathrm{x}$ with TBST, then probed overnight with 1/3000 mouse anti-RBD primary $\mathrm{mAb}$ (Abcam ab277628) solution at $4{ }^{\circ} \mathrm{C}$. Membranes were then washed $5 \mathrm{x}$ with TBST and probed with 1/5000 FITC-labelled goat anti-mouse IgG secondary pAb (Abcam ab6785) and imaged for fluorescence on the Typhoon FLA7000 biomolecular imager (GE healthcare).

\section{Enzyme peptidase and inhibition assays}

Peptidase assays with fluorogenic Boc-Gln-Ala-Arg-AMC substrate (Bachem Cat \# 4017019.0025) were performed in 96-well plates (Greiner Fluotrak) at $200 \mu \mathrm{L}$ reaction volumes in a FlexStation microplate reader (Molecular Devices) at $24^{\circ} \mathrm{C}$. Fluorescence was monitored with the fastest kinetic read settings across 5 minutes at 341:441 nm excitation:emission and converted to a product AMC concentration using standard curves at each substrate concentration to correct for the inner-filter effect ${ }^{7}$ (Extended Data Fig. 4d). All assays contained 2\% (v/v) DMSO and initial reaction velocities were tabulated over the linear portion of the first 60 seconds of progress curves.

To determine Michaelis Menten kinetic parameters, $50 \mu \mathrm{L} 4 \mathrm{x}$ enzyme stock was added through automated addition to microplates containing $150 \mu \mathrm{L}$ substrate $(0.5-1000 \mu \mathrm{M})$ in triplicate and initial reaction velocities were plotted against substrate concentration and curve fit using GraphPad Prism.

Half-maximal inhibitor ( $\mathrm{IC}_{50}$ ) potencies of nafamostat mesylate (MedChemExpress Cat \# HYB0190A), camostat mesylate (MedChemExpress Cat \# HY-13512), benzamidine HCl (Sigma Cat \# 434760-25G), bromhexine $\mathrm{HCl}$ (SelleckChem Cat \# S2060), and SFTI-1 were initially determined by pre-incubating dasTMPRSS2 with inhibitor at concentrations ranging from $0.1 \mathrm{nM}$ $-100 \mu \mathrm{M}$ for $10 \mathrm{~min}$, then enzyme-inhibitor mixes were added to substrate through automated addition. Then, 7 inhibitor concentrations spanning three orders of magnitude across the $\mathrm{IC}_{50}$ value were used and inhibitor reaction velocities were normalized to uninhibited enzyme and plotted as one-site dose response curves in GraphPad. The apparent $\mathrm{Ki}\left(\mathrm{Ki}^{*}\right)$ of classical competitive trypsinlike serine protease inhibitors benzamidine and SFTI-1 were determined using Equation 1,

\begin{tabular}{|l|l|l|}
\hline & $K_{\mathrm{i}}^{*} \approx \frac{\mathrm{IC}_{50}}{1+\frac{[\mathrm{S}]}{K_{\mathrm{M}}}}$ & \\
\hline
\end{tabular}

Where $[\mathrm{S}]$ is the concentration of substrate Boc-QAR-AMC and $K_{\mathrm{M}}$ is the Michaelis constant.

Time-dependent $\mathrm{IC}_{50}$ measurement and $\boldsymbol{k}_{\text {inact }} / K_{\mathrm{i}}$ determination

575

576 inhibitor and nafamostat between $0.01-100 \mathrm{nM}$ with a DMSO control as described. The time dependence of inhibitor potencies was measured by using Flexstation Flex kinetic reads that automatically transferred dasTMPRSS2-inhibitor mixes to substrate wells at the indicated preincubation timepoints (Extended Data Fig. 6c). For the 10s timepoint, a kinetic read was performed after manual addition of enzyme, followed by substrate, using a multichannel pipette. 
Kinetic parameters Kiapp and kinact were determined with the simplified Equations 2 and 3, respectively, assuming a one-step kinetic inhibition mechanism, $\mathrm{A}^{8}$.

\begin{tabular}{|l|c|c|}
\hline Mechanism A : & $\mathrm{E}+\mathrm{I} \stackrel{k_{1}}{\rightarrow} \mathrm{EI}$ & \\
\hline & $K_{\mathrm{i}}^{*} \approx \frac{t_{50}^{(2)}-t_{50}^{(1)}}{t_{50}^{(2)}}-\frac{t_{50}^{(1)}}{I_{50}^{(2)}}$ & \\
\hline & $k_{\text {inact }} \approx \frac{1}{t_{50}^{(2)}} \exp \left[\ln \left(\frac{K_{\mathrm{i}}^{*}}{I_{50}^{(2)}}-1\right)+b\right]$, & (3) \\
\hline
\end{tabular}

585

586

587

588

589

590

591

592

593

594

595

596

597

598

599

600

601

602

603

604

605

606

607

608

609

610

611

612

613

614

615

616

617

where the conversion factor $b=0.558$ is applied for concentrations in $\mu \mathrm{M}$ and time in s.

\section{Active site quantification of dasTMPRSS2}

The acylation of dasTMPRSS2 by nafamostat and concomitant production of the fluorogenic 6amidino-2-napthol leaving group was measured by incubating serial $\log 2$ enzyme dilutions from 6.4-0.8 $\mathrm{nM}$ with excess $(10 \mu \mathrm{M})$ nafamostat, similar to previous efforts with matriptase ${ }^{9}$ (Extended Data Fig. 4e-f). Microplate reading at 320: $490 \mathrm{~nm}$ excitation:emission were used to calculate the number of dasTMPRSS2 active site residues and calibrate peptidase activity and inhibition assays.

\section{Nafamostat inhibition half-life}

The half-life of the phenylguanidino acyl-enzyme complex after nafamostat treatment was measured for dasTMPRSS2 using methods established for camostat with enteropeptidase ${ }^{10}$. Briefly, dasTMPRSS2 $(3.2 \mu \mathrm{M})$ was mixed with slight excess nafamostat ( $5 \mu \mathrm{M}$ or DMSO control) and incubated at room temperature for 20 minutes. After incubation, unbound nafamostat was removed by passage and 3x washes in a $3 \mathrm{kDa}$ MWCO Amicon filter centrifuged at maximum speed. Acylated and untreated dasTMPRSS2 samples were then transferred in quadruplet to a microplate containing either $125 \mu \mathrm{M}$ or $250 \mu \mathrm{M}$ substrate (final concentration of $3.2 \mathrm{nM}$ enzyme). Fluorescent reads were carried out immediately, analogous to $\mathrm{IC}_{50}$ assays, but across a period of 8 hours. The acylated traces were fit to a one-phase association exponential in GraphPad to derive the half-life for activity recovery, normalized to the uninhibited initial reaction velocity.

\section{Differential Scanning Fluorimetry}

Apparent melting temperature $\left(T_{M, a}\right)$ shifts were measured for various dasTMPRSS2-inhibitor coincubations using SYPRO Orange dye (Life Technologies; cat. S-6650) and monitoring fluorescence at 470:510 nm excitation: emission using the Light Cycler 480 II (Roche Applied Science). Samples were prepared in triplicate in 384 well plates (Axygen; Cat\# PCR-384-C; Cat\# UC500) at a final volume of $20 \mu \mathrm{L}$ containing $0.05 \mathrm{mg} / \mathrm{mL}$ dasTMPRSS $2,1 \mu \mathrm{M}$ compound or vehicle control, and 5X SYPRO Orange. Thermal melt curves were generated between $25^{\circ} \mathrm{C}$ to $95^{\circ} \mathrm{C}$ at a gradient of $1{ }^{\circ} \mathrm{C} / \mathrm{min}$ and plots prepared with the DSFworld application ${ }^{11}$ for $T_{M, a}$ determination.

\section{SARS-CoV-2 S protein activation and inhibition}


618 Recombinant SARS-CoV-2 S protein constructs HexaFurin and HexaPro were concentrated to

$6190.5 \mathrm{mg} / \mathrm{mL}$ in Assay Buffer and incubated with the indicated concentrations of furin protease

620 (NEB) or dasTMPRSS2. Digestions took place over 16 hours for furin and from 5-120 minutes

621 for dasTMPRSS2. Furin digestions were terminated by the addition of $4 \mathrm{mM}$ EDTA whereas

622 dasTMPRSS2 digestions were terminated with $5 \mu \mathrm{M}$ nafamostat, then SDS-PAGE samples were

623 immediately prepared with the addition of 4X SDS-PAGE loading buffer and boiled for 5 min at

$62495{ }^{\circ} \mathrm{C} .4 \mu \mathrm{g} \mathrm{S}$ protein were loaded per well under each conditions and gels visualized by

625 Coomassie blue staining. For anti-RBD western blotting, $2 \mu \mathrm{g}$ S protein were loaded per well.

626 For cleavage inhibition assays, dasTMPRSS2 diluted to $320 \mathrm{nM}$ in Assay Buffer was pre-

627 incubated 15 minutes with inhibitor (final 1\% DMSO (v/v)) or DMSO control, then assays were

628 started by transfer of enzyme:inhibitor mixes to S protein. S protein:protease mixtures were

629 incubated at room temperature for 2 hours with nafamostat and 30 minutes for bromhexine.

630

631

632

633

634

635

636

\section{Multiple Sequence Alignments}

Multiple sequence alignments were prepared to compare the human TTSP family members and TMPRSS2 mammalian orthologs. Human TTSP FASTA sequences (isoform 1) were accessed from UniProt and TMPRSS2 orthologs identified with UniProt BLAST. Sequences were aligned with Clustal Omega ${ }^{12}$ and annotated with ESPript $3.0^{13}$.

\section{Protein Visualization and Property Calculation}

The structure of dasTMPRSS2 was inspected and compared to other TTSPs using PyMol (Schrodinger) and the Molecular Operating Environment (MOE; Chemical Computing Group) software suite. The exposed hydrophobic patches of TMPRSS2 were calculated using the MOE Protein Patch Analyzer tool ${ }^{14,15}$.

1. Hsieh, C. et al. Structure-based design of prefusion-stabilized SARS-CoV-2 spikes. 1505, 1501-1505 (2020).

2. Kabsch, W. XDS. Acta Crystallogr. D66, 125-132 (2010).

3. Mccoy, A. J. et al. Phaser crystallographic software. J. Appl. Crystallogr. 40, 658-674 (2007).

4. Emsley, P. \& Lohkamp, B. Features and development of Coot. Acta Crystallogr. D66, 486-501 (2010).

5. Bricogne, G. et al. Buster refinement. (2010).

6. Williams, C. J. et al. MolProbity : More and better reference data for improved all-atom structure validation. Protein Sci. 27, 293-315 (2017).

7. Liu, Y. et al. Use of a fluorescence plate reader for measuring kinetic parameters with inner filter effect correction. Anal. Biochem. 267, 331-335 (1999).

8. Kuzmi , P. A two-point IC 50 method for evaluating the biochemical potency of irreversible enzyme inhibitors. bioRxiv (2020) doi:https://doi.org/10.1101/2020.06.25.171207.

9. Nimishakavi, S., Raymond, W. W., Gruenert, D. C. \& Caughey, G. H. Divergent inhibitor susceptibility among airway lumen-accessible tryptic proteases. PLoS One 10, 1-17 (2015).

10. Sun, W. et al. Targeting Enteropeptidase with Reversible Covalent Inhibitors To Achieve Metabolic Benefits. 510-521 (2020) doi:10.1124/jpet.120.000219. 
664 11. Wu, T. et al. Three Essential Resources to Improve Differential Scanning Fluorimetry ( 665 DSF ) Experiments. BioRxiv (2020).

666 12. Madeira, F. et al. The EMBL-EBI search and sequence analysis tools APIs in 2019.

667

668 Nucleic Acids Res. 47, W636-W641 (2019).

13. Robert, X. \& Gouet, P. Deciphering key features in protein structures with the new ENDscript server. Nucleic Acids Res. 42, W320-W324 (2014).

671

672

14. Jetha, A. et al. Homology modeling and structure-based design improve hydrophobic

673

674

675 interaction chromatography behavior of integrin binding antibodies. MAbs 10, 890-900

676

677

678

679

680

681

682

683

684

685

686

687

688

689

690

691

692

693

694

695

696

697

698

699

700

701

702

703

704

705

706

707

708

709 